\title{
On the symmetric polynomials in the variety of Grassmann algebras
}

\author{
Nazan AKDOĞAN ${ }^{1}$ \\ ${ }^{1}$ İstanbul Teknik Üniversitesi, Fen Edebiyat Fakültesi, Matematik Bölümü, İstanbul, Türkiye \\ Çukurova Üniversitesi, Fen Edebiyat Fakültesi, Matematik Bölümü, Adana, Türkiye
}

Geliş / Received: 05/05/2020, Kabul / Accepted: 15/09/2021

\begin{abstract}
Let $K$ be a field of characteristic zero and $L$ be the associative algebra of rank 2 over $K$ in the variety generated by Grassmann algebras. In this paper, we study the subalgebra $L^{S_{2}}$ of symmetric polynomials in the algebra $L$, and give a finite generating set for $L^{S_{2}}$.
\end{abstract}

Keywords: PI-algebra, Grassmann algebras, symmetric polynomial

\section{Grassmann cebirleri sınıfinda simetrik polinomlar üzerine}

Öz

$K$ karakteristiği sıfir olan bir cisim ve $L$, Grassmann cebirleri tarafindan üretilen varyetede, $K$ cismi üzerinde rankı 2 olan birleşmeli cebir olsun. Bu çalışmada, $L$ cebirinin $L^{S_{2}}$ simetrik polinomlar alt cebiri incelenmiş ve $L^{S_{2}}$ için sonlu bir üreteç kümesi verilmiştir.

Anahtar Kelimeler: PI-cebiri, Grassmann cebirleri, simetrik polinom.

\section{Introduction}

Let $A_{n}$ be a free unitary associative algebra generated by $x_{1}, \ldots, x_{n}$ over a field $K$ of characteristic zero. The Grassmann algebra is the factor algebra $G_{n}=A_{n} / I$ where $I$ is generated by $x_{i} x_{j}+x_{j} x_{i}, 1 \leq i, j \leq n$. The Grassmann algebra is generated by $e_{i}=x_{i}+I, 1 \leq i \leq n$, which implies that $e_{i} e_{j}+e_{j} e_{i}=0$. As a vector space, the Grassmann algebra has the basis $B=\left\{e_{i_{1}} \ldots e_{i_{k}}: i_{1} \leq \cdots \leq i_{k}, 1 \leq k \leq n\right\} \cup\{1\}$.

Let $K\langle X\rangle$ be the free associative algebra generated by $X$ over $K$ where $X=\left\{x_{1}, x_{2}, \ldots\right\}$ is a countable infinite set of variables. We call elements of $K\langle X\rangle$ polynomials. Let $A$ be an algebra over $K$ and $f\left(x_{1}, \ldots, x_{n}\right) \in K\langle X\rangle$. We call $f\left(x_{1}, \ldots, x_{n}\right)$ a polynomial identity of $A$, if $f\left(a_{1}, \ldots, a_{n}\right)=0$ for all $a_{1}, \ldots, a_{n} \in A$. The algebra $A$ is called a PI-algebra if it has a nontrivial 
polynomial identity. We denote by $T(A)$ the set of all polynomial identities of $A$. Since $T(A)$ is an ideal of $K\langle X\rangle$ which is invariant under all endomorphisms of $K\langle X\rangle$, it is a T-ideal of $A$.

One of the objectives of the theory of $P I$-algebras is finding the generating sets for $T$-ideal of an algebra. Given a commutative unitary algebra, the $T$-ideal of the algebra is generated by the commutator $[x, y]=x y-y x$. Since $[[x, y], z]=0$ for all $x, y, z \in G_{n}$, the Grassmann algebra $G_{n}$ is a $P I$-algebra. It is shown that the $T$-ideal of the Grassmann algebras is generated by $[[x, y], z]$. It is shown by Latyshev (1976) and by Krakovski and Regev (1973).

The variety defined by the polynomial identity $[[x, y], z]=0$ from $T\left(G_{n}\right)$ is called the variety generated by the Grassmann algebra. Let us denote by $L$ the free associative algebra of rank 2 generated by $\{x, y\}$ in the variety generated by the Grassmann algebra.

In this paper, we investigate the subalgebra of symmetric polynomials in the algebra $L$. We give a generating set for the algebra of symmetric polynomials as an algebra and obtain the presentation of the commutator ideal of the algebra of symmetric polynomials.

\section{Preliminaries}

Let $K$ be a field of characteristic zero, $K\left[x_{1}, \ldots, x_{n}\right]$ be the commutative algebra of polynomials. A polynomial $f\left(x_{1}, \ldots, x_{n}\right) \in K\left[x_{1}, \ldots, x_{n}\right]$ is symmetric if it is invariant under every permutation of the variables $x_{1}, \ldots, x_{n}$.

The polynomials $\sigma_{1}, \ldots, \sigma_{n} \in K\left[x_{1}, \ldots, x_{n}\right]$ are called the elementary symmetric polynomials, where

$$
\begin{gathered}
\sigma_{1}=x_{1}+\cdots+x_{n} \\
\sigma_{2}=x_{1} x_{2}+x_{1} x_{3}+\cdots+x_{2} x_{3}+\cdots+x_{n-1} x_{n} \\
\sigma_{3}=x_{1} x_{2} x_{3}+x_{1} x_{2} x_{4}+\cdots+x_{n-2} x_{n-1} x_{n} \\
\vdots \\
\sigma_{n}=x_{1} x_{2} x_{3} \cdots x_{n} .
\end{gathered}
$$

The elementary symmetric polynomials are generators of the algebra of symmetric polynomials. Every symmetric polynomial can be written uniquely as a polynomial in the elementary symmetric polynomials. The generating set is not unique. The polynomials 
$p_{1}, \ldots, p_{n} \in K\left[x_{1}, \ldots, x_{n}\right]$ form another generating set for the symmetric polynomials, where $p_{k}=x_{1}^{k}+\cdots+x_{n}^{k}$. (see Cox et al., 2015; Strumfels, 2008; van der Waerden, 1949.)

We refer the readers to the work by Fındık and Öğüşlü (2019) for a generating set of symmetric polynomials in the free metabelian Lie algebra of rank 2 as one of the generalizations in a Lie algebraic setting.

Now let $L$ be the algebra of rank 2 freely generated by elements $x, y$, in the variety generated by the Grassmann algebra consisting of unitary associative algebras over the ground field $K$. The ideal $I$ of $L$ generated by all commutators $[r, s]=r s-s r$, where $r, s \in L$, is called the commutator ideal of $L$. The elements of $I$ are of the form $\sum \alpha p[r, s] t$ where $p, t \in L, \alpha \in K$. It is well known (see e.g. Drensky (1996) ) that the basis of the commutator ideal $I$ as a vector space consists of elements $x^{a} y^{b}[x, y], a, b \geq 0$. The commutative polynomial algebra $K[x, y]$ acts on $I$ by the following action.

$$
p \sum \alpha_{a b} x^{a} y^{b}[x, y]=\sum \alpha_{a b} p x^{a} y^{b}[x, y]
$$

where $p \in K[x, y]$ and $\sum \alpha_{a b} x^{a} y^{b}[x, y] \in I$. Therefore the commutator ideal $I$ is a free $K[x, y]$ module generated by the commutator $[x, y]$ of $x$ and $y$.

The polynomial identity $[[x, y], z]=0$ implies the identity

$$
[x, y][z, t]=-[x, z][y, t]
$$

and

$$
x^{a} y^{b}[x, y]=y^{b} x^{a}[x, y]=[x, y] x^{a} y^{b}=[x, y] y^{b} x^{a}
$$

is satisfied in $I$.

We define the sets of symmetric polynomials of $L$ and $I$ by

$$
L^{S_{2}}=\{p(x, y) \in L: p(x, y)=p(y, x)\}
$$

and

$$
I^{S_{2}}=\{p(x, y) \in I: p(x, y)=p(y, x)\}
$$

respectively. These sets are subalgebras of invariants of the symmetric group $S_{2}$. 


\section{Results and Discussion}

Lemma 3.1. Let $p(x, y)$ be an element in $I^{S_{2}}$. Then $p(x, y)$ is of the form

$$
p(x, y)=\sum_{0 \leq a<b} \alpha_{a b}\left(x^{a} y^{b}-x^{b} y^{a}\right)[x, y]
$$

for some $\alpha_{a b} \in K$.

Proof. The element $p(x, y) \in I^{S_{2}} \subseteq I$ can be expressed as

$$
p(x, y)=\sum_{0 \leq a, b} \alpha_{a b} x^{a} y^{b}[x, y]=\sum_{a \neq b} \alpha_{a b} x^{a} y^{b}[x, y]+\sum_{0 \leq a} \alpha_{a a} x^{a} y^{a}[x, y]
$$

Since $p(x, y) \in I^{S_{2}}, p(x, y)=p(y, x)$ holds. Hence

$$
p(x, y)=\sum_{a \neq b} \alpha_{a b} y^{a} x^{b}[y, x]+\sum_{0 \leq a} \alpha_{a a} y^{a} x^{a}[y, x]
$$

and

$$
\sum_{0 \leq a} 2 \alpha_{a a} x^{a} y^{a}[x, y]+\sum_{a \neq b} \alpha_{a b} x^{a} y^{b}[x, y]-\sum_{a \neq b} \alpha_{a b} y^{a} x^{b}[y, x]=0
$$

Since $\sum_{0 \leq a} 2 \alpha_{a a} x^{a} y^{a}[x, y]=0$ by the suggested basis, we have $\alpha_{a a}=0$ for $0 \leq a$. Therefore we have

$$
\sum_{a<b}\left(\alpha_{a b}+\alpha_{b a}\right) x^{a} y^{b}[x, y]+\sum_{b<a}\left(\alpha_{a b}+\alpha_{b a}\right) x^{a} y^{b}[x, y]=0
$$

where each sum equals zero by linear independence. So that $\alpha_{a b}=-\alpha_{b a}$ for all $a \neq b$. Thus we have the following computations which provide the desired form of the element $p(x, y)$.

$$
\begin{gathered}
p(x, y)=\sum_{a<b} \alpha_{a b} x^{a} y^{b}[x, y]+\sum_{b<a} \alpha_{a b} x^{a} y^{b}[x, y] \\
=\sum_{a<b} \alpha_{a b} x^{a} y^{b}[x, y]+\sum_{a<b} \alpha_{b a} x^{b} y^{a}[x, y] \\
=\sum_{0 \leq a<b} \alpha_{a b}\left(x^{a} y^{b}-x^{b} y^{a}\right)[x, y] .
\end{gathered}
$$


Corollary 3.2. The set

$$
\left\{\left(x^{a} y^{b}-x^{b} y^{a}\right)[x, y]: 0 \leq a<b\right\}
$$

is the basis of $I^{S_{2}}$.

Proof. The given set spans $I^{S_{2}}$ as a vector space by Lemma 3.1. It is sufficient to show that the set is linearly independent. Let

$$
\sum_{a<b} \alpha_{a b}\left(x^{a} y^{b}-x^{b} y^{a}\right)[x, y]=0
$$

We can fix $a+b$ to $n$ since $L^{S_{2}}$ is a graded vector space.

$$
\sum_{a+b=n, a<b} \alpha_{a b}\left(x^{a} y^{b}-x^{b} y^{a}\right)[x, y]=0
$$

As $I$ is the $K[x, y]$-module generated by $[x, y]$, we have

$$
\sum_{a+b=n, 0 \leq a<b} \alpha_{a b} x^{a} y^{b}-\sum_{a+b=n, 0 \leq a<b} \alpha_{a b} x^{b} y^{a}=0 .
$$

So $\alpha_{a b}=0$ where $0 \leq a<b$ since each sum equals zero.

Theorem 3.3. The presentation of $I^{S_{2}}$ is

$$
I^{S_{2}}=\left\langle m_{\boldsymbol{a} \boldsymbol{b}} \mid 0 \leq a<b, m_{\boldsymbol{a b}} m_{\boldsymbol{a}^{\prime} \boldsymbol{b}^{\prime}}=0\right\rangle
$$

where $m_{\boldsymbol{a b}}=\left(x^{a} y^{b}-x^{b} y^{a}\right)[x, y]$.

Proof. Let $m_{\boldsymbol{a}^{\prime} \boldsymbol{b}^{\prime}}=\left(x^{\boldsymbol{a}^{\prime}} y^{\boldsymbol{b}^{\prime}}-x^{\boldsymbol{b}^{\prime}} y^{\boldsymbol{a}^{\prime}}\right)[x, y]$.

$$
\begin{aligned}
& m_{\boldsymbol{a} \boldsymbol{b}} m_{\boldsymbol{a}^{\prime} \boldsymbol{b}^{\prime}}=\left(x^{a} y^{b}-x^{b} y^{a}\right)[x, y]\left(x^{\boldsymbol{a}^{\prime}} y^{\boldsymbol{b}^{\prime}}-x^{\boldsymbol{b}^{\prime}} y^{\boldsymbol{a}^{\prime}}\right)[x, y] \\
& =\left(x^{a} y^{b}-x^{b} y^{a}\right)\left(x^{\boldsymbol{a}^{\prime}} y^{\boldsymbol{b}^{\prime}}-x^{\boldsymbol{b}^{\prime}} y^{\boldsymbol{a}^{\prime}}\right)[x, y][x, y] \\
& =\left(x^{a} y^{b}-x^{b} y^{a}\right)\left(x^{\boldsymbol{a}^{\prime}} y^{\boldsymbol{b}^{\prime}}-x^{\boldsymbol{b}^{\prime}} y^{\boldsymbol{a}^{\prime}}\right)(x y-y x)[x, y] \\
& =\left(x^{a} y^{b}-x^{b} y^{a}\right)\left(x^{\boldsymbol{a}^{\prime}} y^{\boldsymbol{b}^{\prime}}-x^{\boldsymbol{b}^{\prime}} y^{\boldsymbol{a}^{\prime}}\right)(x y)[x, y]-\left(x^{a} y^{b}-x^{b} y^{a}\right)\left(x^{\boldsymbol{a}^{\prime}} y^{\boldsymbol{b}^{\prime}}-x^{\boldsymbol{b}^{\prime}} y^{\boldsymbol{a}^{\prime}}\right)(x y)[x, y] \\
& =0 .
\end{aligned}
$$

Theorem 3.4. The algebra $L^{S_{2}}$ is generated by the set $\left\{x+y, x^{2}+y^{2},(y-x)[x, y]\right\}$, and the algebra $I^{S_{2}}$ is a left $K[x, y]^{S_{2}}$-module generated by the element $(y-x)[x, y]$. 
Proof. The algebra $(L / I)^{S_{2}} \cong K[x, y]^{S_{2}}$ is generated by $x+y, x^{2}+y^{2}$. Thus it is sufficient to show that the algebra $I^{S_{2}}$ is contained in the algebra generated by $x+y, x^{2}+y^{2},(y-x)[x, y]$. Corollary 3.2 gives that $I^{S_{2}}$ has the basis

$$
\left\{\left(x^{a} y^{b}-x^{b} y^{a}\right)[x, y]: 0 \leq a<b\right\}
$$

which can be also considered as generating set. Direct computations give that

$$
\left(x^{a} y^{b}-x^{b} y^{a}\right)[x, y]=q(x, y) r(x, y)(y-x)[x, y]
$$

where

$$
q(x, y)=\left(\frac{(x+y)^{2}-\left(x^{2}+y^{2}\right)}{2}\right)^{a}
$$

and

$$
r(x, y)=\sum_{i=1}^{b-a} x^{b-a-i} y^{i-1}
$$

The polynomial $r(x, y)$ can be written as $r(x, y)=\frac{x^{b-a}-y^{b-a}}{x-y}$. It is clear that $r(x, y)=r(y, x)$ and $q(x, y)=q(y, x)$. Hence $q(x, y), r(x, y) \in K\left[x+y, x^{2}+y^{2}\right]$, and $\left(x^{a} y^{b}-x^{b} y^{a}\right)[x, y]$ is included in $\left\langle x+y, x^{2}+y^{2},(y-x)[x, y]\right\rangle$. This also shows that $I^{S_{2}}$ is a left $K\left[x+y, x^{2}+y^{2}\right]=K[x, y]^{S_{2}}$-module.

\section{References}

Cox, D., Little, J. and O’Shea, D. (2015). “Ideals Varieties, and Algorithms 4th ed.”, Springer, New York, 345-352.

Drensky, V. (1996). "Free Algebras and PI-Algebras”, Springer, Singapore, 12-51.

Find1k, Ş. and Öğüşlü, N.Ş. 2019. "Palindromes in the free metabelian Lie algebras", Int. J. Algebra Comput., 29(5), 885-891.

Krakovski, D. and Regev, A. 1973. "The Polynomial Identities of the Grassmann Algebra", Trans. Amer. Math. Soc., 181, 429-438.

Latyshev, V.N., 1976. "Partially Ordered Sets and Nonmatrix Identities of Associative Algebras" Algebr. Log., 15(1), 34-45. 
Strumfels, B. (2008). “Algorithms in Invariant Theory 2nd ed.”, Springer-Verlag, Wien, 2-6. van der Waerden, B.L. (1949). "Modern Algebra”, F. Ungar, New York, 78-82. 